\title{
ANALYZING THE BINARY PULSARS ABOVE THE SPIN-UP LINE
}

\author{
YUANYUE PAN ${ }^{*, \dagger, \ddagger}$, NA WANG* $^{*}$ and CHENGMIN ZHANG ${ }^{\dagger}$ \\ *Xinjiang Astronomical Observatory, Chinese Academy of Sciences \\ Xinjiang 830011, China \\ ${ }^{\dagger}$ National Astronomical Observatories, Chinese Academy of Sciences \\ Beijing 100012, China \\ ‡panyuanyue@xao.ac.cn
}

\begin{abstract}
With accretion of mass from an evolving companion star, a neutron star can be spun up to an equilibrium period, which is set by the Kepler orbital period at the magnetosphere radius, expressed as a proportional relation between the spin period and magnetic field strength. The spin-up line relationship can be also expressed as a relation between period $(P)$ and period derivative $(\dot{P})$ for the pulsars with dipole radiation. All binary pulsars should lie below the spin-up line, if they accrete the sufficient masses from companions. So, we investigate the distribution of isolated and binary pulsars in the period $(P)$ and magnetic field $(B)$ diagram to see their positions relative to the spin-up line. It is found that the eight binary pulsars (of total 172) are above the spin-up line, thus we analyze their properties and present the possible process of their formations.
\end{abstract}

Keywords: Neutron star; binary pulsar; spin-up line.

\section{Introduction}

A pulsar is classified on the basis of its observed properties: its spin period and period derivation. ${ }^{1}$ These properties can give us an estimate of the pulsar surface magnetic field and a characteristic age. Researchers always divided the neutron stars(NSs) into two kinds according to their surface magnetic field and spin period. One group is the normal pulsars with magnetic field $B \sim 10^{12} \mathrm{G}$ and spin period $P \sim 0.5 s$. The other group is millisecond pulsars with magnetic field $B \sim 10^{8.5}$ and spin period $P \sim 10 \mathrm{~ms}^{2-6}$ It was found that the majority of millisecond pulsars are always in binary system. Then in the binary system, the NS fields decay, while experienced accretion, ${ }^{7}$ mechanism of which has been considered as the accretion flow to drag the field lines aside to dilute the polar field strength. ${ }^{8}$ When a neutron star gets to its minimum spin period to which such a spin up proceeds in an Eddington-limited accretion, we can get the spin-up line. The equation of the spin-up line can be written as: ${ }^{2}$

$$
B_{9}^{\frac{6}{7}}=\frac{P}{2.2} R_{6}^{-\frac{18}{7}} m^{\frac{8}{7}} \times 10^{3}
$$


or expressed by the period derivative as,

$$
P_{\min }^{\frac{4}{7}} \dot{p}^{-\frac{3}{7}} \simeq 0.8 R_{6}^{\frac{18}{7}} m^{-\frac{8}{7}}
$$

where $B_{9}$ is the magnetic field in units of $10^{9} G, R_{6}$ is the stellar radius in units of $10^{6} \mathrm{~cm}, m$ is in unit of solar mass. The mass range of NSs can be from $1 M_{\odot}$ to $3.2 M_{\odot} \cdot{ }^{9}$ In binary system, the average mass for millisecond pulsars and others are, respectively, $M=1.57 \pm 0.35 M_{\odot}$ and $M=1.37 \pm 0.23 M_{\odot} \cdot{ }^{10}$ In order to get the spin-up lien in the figure, we choose $R_{6}=1$ and $m=1.4 M_{\odot}$. All binary pulsars should be under this line if sufficient mass is accreted.

In this paper, we will make a statistics of isolated pulsars and binary pulsars using the data from ATNF pulsar catalogue (see http://www.atnf.csiro.au/). Then, we will focus on eight special binary pulsars that are above the spin-up line, and deduce their possible formation pathes. Finally, the summary and discussion will be given.

\section{Statistics of Isolated Neutron Stars and Binary Pulsars}

Figure 1 shows us the distribution of isolated pulsars and binary pulsars. The solid line in the Fig. 1 is the spin-up line, and the dashed line is the death line. From the Fig. 1(a) we know that lots of isolated pulsars are young pulsars that are above the spin-up line. Fig. 1b shows us during all binary pulsars, there are eight binary pulsars above the spin-up line. In theory, all binaries should lie below the spin-up line, if they accrete the sufficient masses from companions. So, we put the information of these eight binaries in Table1, data from ATNF pulsar catalogue. In the table, we find that none of the binaries is millisecond pulsar because all of their spin periods are larger than $20 \mathrm{~ms} .{ }^{11}$ However, these eight pulsars will go to the recycled process in enough time. The main pulsar will get the mass from its companion. Its surface

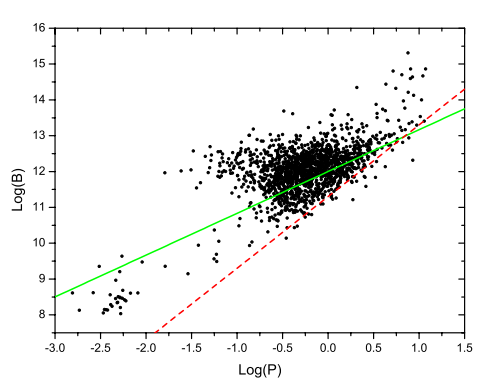

(a) dots stand for isolated pulsars. It includes 1812 single pulsars, where 1632 single pulsars have both $B$ and $P$.

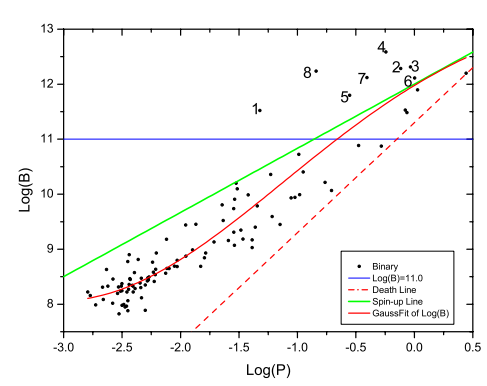

(b) dots stand for binary pulsars. It includes 172 binary pulsars, where 106 binary pulsars have both $B$ and $P$.

Fig. 1. Magnetic field versus spin period diagram, data from ATNF pulsar catalogue. The solid line is the spin-up line and the dashed line is the death line. 
magnetic field will decay, then it will become a recycled pulsar during the recycle process. Its companion will become a neutron star or a white dwarf according to their initial condition. ${ }^{5}$ With the mass of their companions, we put the eight dots into two types:

Type (I): Companion is a massive star whose mass is larger than $2.0 M_{\odot}$ (No. 1 , $2,3,4)$. No.1 is an X-ray pulsar with a Be star as its companion. ${ }^{12}$ The orbit period is so $\operatorname{long}\left(P_{\text {orbit }}=1236.724\right.$ Days $)$ and the eccentricity orbit is so high $(e=0.8699)$ that the pulsar can only get accretion mass from its companion when the companion is near the periastron. It is a new born binary system due to its characteristic age(332000Years). In the future the system will have a recycled NS and its companion will become a low mass neutron star. No.2 is in the cluster from where the pulsar accretes the mass. In the cluster, the pulsar might capture its companion somewhere. Although it has long enough characteristic age(2.53E6Years), the long obit $\operatorname{period}\left(P_{\text {orbit }}=1940.9\right.$ Days $)$ and highly eccentricity $(e=0.955)$ would make the evolution be slowed down. No.3 is a radio pulsar with a B star as its companion. It has highly eccentricity $(e=0.8079)$ which can make it accurate little mass from its companion. It may go into the recycled process soon because of its large characteristic age(3.29E6Years). In the future it may become a recycled pulsar and its companion may be a normal neutron star. No.4 is a new born radio pulsar due to its short characteristic age $(354000$ Y ears $)$, long orbital period $\left(P_{\text {orbit }}=231.0297\right.$ Days $)$ and high eccentricity $(e=0.5789)$. Its final fate may also be a millisecond pulsar in binary pulsar system after the recycle process.

Type (II): Companion is a white dwarf whose mass is less than $1.4 M_{\odot}$ (No. 5 , $6,7,8)$. No.5 is a radio pulsar. ${ }^{13}$ Its long orbital period $\left(P_{\text {orbit }}=357.762\right.$ Days $)$ and highly eccentricity $(e=0.7946)$ will make it difficult to get mass from the companion. No.6 is an optical pulsar which is in the cluster. Science it almost arrives at the spin-up line and it has short orbital period $\left(P_{\text {orbit }}=0.2583\right)$ and low eccentricity $(e=0.0064)$, we can include that it will spend less time than No.3 to achieve its recycle process. No.7 is not in the cluster, but it has short orbital $\operatorname{period}\left(P_{\text {orbit }}=0.1977\right.$ Days $)$ and low eccentricity $(e=0.1719)$ like No.6, so it will also go to its recycled process in short time. No.8 has the shortest orbital period $\left(P_{\text {orbit }}=0.166\right.$ Days $)$ in the eight pulsars and low eccentricity. ${ }^{14}$ However, with its very short characteristic age and a high surface magnetic field $(B=1.73 E 12 G)$, it can be said that No.8 is a new born pulsar and its predecessor might be a magnetar.

\section{Summary and Conclusion}

In this paper, the distribution of the isolated pulsars and binary pulsars is given. For the eight binaries above the spin-up line, we deduced their possible reasons why they are above the spin-up line: (1)If the characteristic age of the pulsar is less than the normal pulsar $\left(\sim 10^{6 \sim 7}\right.$ Years $)$, it is probably a new pulsar that is above the spin-up lien. (2)A binary pulsar with long orbital period and a highly eccentricity 
Table 1. Information of 8 binary pulsars above the spin-up line.

\begin{tabular}{lcccccccc}
\hline No. & Name & $\begin{array}{c}P_{\text {spin }} \\
\text { ms }\end{array}$ & $\begin{array}{c}\text { Binary } \\
\text { Type }\end{array}$ & $\begin{array}{c}P_{\text {ord }} \\
\text { days }\end{array}$ & Ecc & $\begin{array}{c}M_{C} \\
\text { M } \odot\end{array}$ & $\begin{array}{c}\text { Age } \\
\text { Yr }\end{array}$ & $\begin{array}{c}\text { B } \\
\text { G }\end{array}$ \\
\hline 1 & B1259-63 & 47.763 & MSS & 1236.724 & 0.8699 & 4.14 & 332000 & $3.34 \mathrm{E} 11$ \\
2 & J1638-4725 & 763.933 & MSS & 1940.9 & 0.955 & 8.08 & $2.53 \mathrm{E} 6$ & $1.93 \mathrm{E} 12$ \\
3 & J0045-7319 & 926.276 & BT & 51.1695 & 0.8079 & 5.27 & $3.29 \mathrm{E} 6$ & $2.06 \mathrm{E} 12$ \\
4 & J1740-3052 & 570.31 & MSS & 231.0297 & 0.5789 & 15.82 & 354000 & $3.86 \mathrm{E} 12$ \\
\hline 5 & B1820-11 & 279.829 & BT & 357.762 & 0.7946 & 0.78 & $3.22 \mathrm{E} 6$ & $6.29 \mathrm{E} 11$ \\
6 & B1718-19 & 1004.037 & ELL1 & 0.2583 & 0.0064 & 0.13 & $9.8 \mathrm{E} 6$ & $1.29 \mathrm{E} 12$ \\
7 & J1141-6545 & 393.899 & DDGR & 0.1977 & 0.1719 & 1.21 & $1.45 \mathrm{E} 6$ & $1.32 \mathrm{E} 12$ \\
8 & J1906+0746 & 144.072 & BT & 0.166 & 0.0853 & 0.98 & 113000 & $1.73 \mathrm{E} 12$ \\
\hline
\end{tabular}

can only get mass when it goes to the place near the periastron. So the pulsar with long orbital period and high eccentricity will have a long time to get recycle. (3)Normal pulsar always has surface magnetic field around $10^{12} G$. When we infer whether the binary system is a young one, it should be taken the three conditions together. Beyond these three conditions, it also should be considered whether the binary system is in the cluster. If the pulsar is in the cluster, it will take time to capture its company somewhere.

\section{Acknowledgments}

This research has been supported by NSFC (No.10773034), CAS Knowledge Innovation Project (KJCX2-YW-T09) and NBRPC(2009CB824800).

\section{References}

1. I. H. Stairs, Science 304 (2004) 23.

2. D. Bhattacharya and E. P. J. van den Heuvel, Phys. Rep. 203 (1991) 1.

3. D. R. Lorimer, Living Rev. Relativity 11 (2008) 8.

4. R. N. Manchester, G. B. Hobbs, A. Teoh and M. Hobbs AJ 129(2005) 1993.

5. J. Wang, C. M. Zhang, Y. H. Zhao et al., A\&A 526 (2011) 88.

6. M. A. Alpar, A. F. Cheng, M. A. Ruderman et al.,Nature 300 (1982) 728.

7. R. E. Taam and E. P. J. Van den Heuvel, ApJ 305 (1986) 235.

8. C. M. Zhang and Y. Kojima, MNRAS 336 (2006) 137.

9. C. E. Rhoades, R. Ruffini, Physical Review Letters 1 (1974)324.

10. C. M. Zhang, J. Wang, Y. H. Zhao et al., A\&A 527, (2011) 83.

11. E. P. J. van den Heuvel, Science 303 (2004) 20.

12. N. Wang, S. Johnston, R. N. Manchester et al., MNRAS 351 (2004) 599.

13. I. H. Stairs, MNRAS 325 (2001) 979.

14. D. R. Lorimer, I. H. Stairs, P. C. Freire et al., APJ 640 (2006) 428. 